Supporting Information

\title{
Thermodynamic modeling of in situ leaching of sandstone- type uranium minerals
}

Nao SHEN ${ }^{1,2}$, Jun $\mathrm{LI}^{1 *}$, Yongfan GUO ${ }^{1,2}$, Xiaochun LI ${ }^{1}$

${ }^{1}$ State Key Laboratory of Geomechanics and Geotechnical Engineering, Institute of Rock and Soil Mechanics, Chinese Academy of Sciences, Wuhan 430071, China

${ }^{2}$ University of Chinese Academy of Sciences, Beijing 100049, China

*Corresponding author: Jun LI

E-mail address: lijun2009s@gmail.com (J. LI). 
Table S1. Complexation reactions of uranium

\begin{tabular}{|c|c|c|c|c|}
\hline Reaction & $\log K$ & $\Delta_{r} H_{m}^{0}(\mathrm{~kJ} / \mathrm{mol})$ & $\dot{a} a$ & $\operatorname{Ref}^{b}$ \\
\hline $\mathrm{U}^{+4}$ & & & 5.5 & \\
\hline $\mathrm{U}^{+4}+\mathrm{H}_{2} \mathrm{O} \rightleftharpoons \mathrm{UOH}^{+3}+\mathrm{H}^{+}$ & -0.533 & 46.808 & 5 & 2 \\
\hline $\mathrm{U}^{+4}+\mathrm{H}_{2} \mathrm{O} \rightleftharpoons \mathrm{UO}^{+2}+2 \mathrm{H}^{+}$ & -1.999 & 73.139 & 4.5 & 1 \\
\hline $\mathrm{U}^{+4}+2 \mathrm{H}_{2} \mathrm{O} \rightleftharpoons \mathrm{HUO}_{2}^{+}+3 \mathrm{H}^{+}$ & -4.988 & 96.790 & 4.0 & 1 \\
\hline $\mathrm{U}^{+4}+4 \mathrm{OH}^{-} \rightleftharpoons \mathrm{U}(\mathrm{OH})_{4}(\mathrm{aq})$ & 46.0 & -113.347 & 3.0 & 3 \\
\hline $\mathrm{U}^{+4}+\mathrm{Cl}^{-} \rightleftharpoons \mathrm{UCl}^{+3}$ & 1.7073 & -18.9993 & 5.0 & 2 \\
\hline $\mathrm{U}^{+4}+2 \mathrm{SO}_{4}^{-2} \rightleftharpoons \mathrm{U}\left(\mathrm{SO}_{4}\right)_{2}(\mathrm{aq})$ & 10.3507 & 33.2232 & 3.0 & 2 \\
\hline $\mathrm{U}^{+4}+\mathrm{SO}_{4}^{-2} \rightleftharpoons \mathrm{USO}_{4}^{+2}$ & 6.5003 & 8.2616 & 4.5 & 2 \\
\hline $\mathrm{UO}_{2}^{+2}$ & & & 4.5 & \\
\hline $\mathrm{UO}_{2}^{+2}+\mathrm{H}_{2} \mathrm{O} \rightleftharpoons \mathrm{UO}_{2} \mathrm{OH}^{+}+\mathrm{H}^{+}$ & -5.25 & 43.459 & 4.0 & 3 \\
\hline $\mathrm{UO}_{2}{ }^{+2}+2 \mathrm{H}_{2} \mathrm{O} \rightleftharpoons \mathrm{UO}_{2}(\mathrm{OH})_{2}(\mathrm{aq})+2 \mathrm{H}^{+}$ & -12.15 & 0 & 3.0 & 3 \\
\hline $\mathrm{UO}_{2}^{+2}+3 \mathrm{H}_{2} \mathrm{O} \rightleftharpoons \mathrm{UO}_{2}(\mathrm{OH})_{3}^{-}+3 \mathrm{H}^{+}$ & -20.73 & 0 & 4.0 & 4 \\
\hline $\mathrm{UO}_{2}^{+2}+4 \mathrm{H}_{2} \mathrm{O} \rightleftharpoons \mathrm{UO}_{2}(\mathrm{OH})_{4}^{-2}+4 \mathrm{H}^{+}$ & -31.92 & 0 & 4.0 & 4 \\
\hline $2 \mathrm{UO}_{2}^{+2}+\mathrm{H}_{2} \mathrm{O} \rightleftharpoons\left(\mathrm{UO}_{2}\right)_{2} \mathrm{OH}^{+3}+\mathrm{H}^{+}$ & -2.7072 & 0 & 5.0 & 2 \\
\hline $2 \mathrm{UO}_{2}{ }^{+2}+2 \mathrm{H}_{2} \mathrm{O} \rightleftharpoons\left(\mathrm{UO}_{2}\right)_{2}(\mathrm{OH})_{2}{ }^{+2}+2 \mathrm{H}^{+}$ & -5.6346 & 37.613 & 4.5 & 2 \\
\hline $3 \mathrm{UO}_{2}^{+2}+4 \mathrm{H}_{2} \mathrm{O} \rightleftharpoons\left(\mathrm{UO}_{2}\right)_{3}(\mathrm{OH})_{4}^{+2}+4 \mathrm{H}^{+}$ & -11.929 & 0 & 4.5 & 2 \\
\hline $3 \mathrm{UO}_{2}^{+2}+5 \mathrm{H}_{2} \mathrm{O} \rightleftharpoons\left(\mathrm{UO}_{2}\right)_{3}(\mathrm{OH})_{5}^{+}+5 \mathrm{H}^{+}$ & -15.586 & 97.1056 & 4.0 & 2 \\
\hline $3 \mathrm{UO}_{2}^{+2}+7 \mathrm{H}_{2} \mathrm{O} \rightleftharpoons\left(\mathrm{UO}_{2}\right)_{3}(\mathrm{OH})_{7}^{-+}+7 \mathrm{H}^{+}$ & -32.2 & 0 & 4.0 & 3 \\
\hline $4 \mathrm{UO}_{2}^{+2}+7 \mathrm{H}_{2} \mathrm{O} \rightleftharpoons\left(\mathrm{UO}_{2}\right)_{4}(\mathrm{OH})_{7}^{+}+7 \mathrm{H}^{+}$ & -21.951 & 0 & 4.0 & 2 \\
\hline $\mathrm{UO}_{2}{ }^{+2}+\mathrm{Cl}^{-} \rightleftharpoons \mathrm{UO}_{2} \mathrm{Cl}^{+}$ & 0.1572 & 8.0 & 4.0 & 2 \\
\hline $\mathrm{UO}_{2}{ }^{+2}+2 \mathrm{Cl}^{-} \rightleftharpoons \mathrm{UO}_{2} \mathrm{Cl}_{2}(\mathrm{aq})$ & -1.1253 & 15.0013 & 3.0 & 2 \\
\hline $\mathrm{UO}_{2}{ }^{+2}+\mathrm{CO}_{3}^{-2} \rightleftharpoons \mathrm{UO}_{2} \mathrm{CO}_{3}(\mathrm{aq})$ & 9.94 & 5.0 & 3.0 & 3 \\
\hline $\mathrm{UO}_{2}{ }^{+2}+2 \mathrm{CO}_{3}^{-2} \rightleftharpoons \mathrm{UO}_{2}\left(\mathrm{CO}_{3}\right)_{2}^{-2}$ & 16.61 & 18.5 & 4.0 & 3 \\
\hline $\mathrm{UO}_{2}{ }^{+2}+3 \mathrm{CO}_{3}^{-2} \rightleftharpoons \mathrm{UO}_{2}\left(\mathrm{CO}_{3}\right)_{3}^{-4}$ & 21.84 & -39.2 & 4.0 & 3 \\
\hline $3 \mathrm{UO}_{2}^{+2}+6 \mathrm{HCO}_{3}^{-} \rightleftharpoons\left(\mathrm{UO}_{2}\right)_{3}\left(\mathrm{CO}_{3}\right)_{6}^{-6}+6 \mathrm{H}^{+}$ & -8.0601 & 25.5204 & 4.0 & 2 \\
\hline $2 \mathrm{UO}_{2}{ }^{+2}+\mathrm{HCO}_{3}{ }^{-}+3 \mathrm{H}_{2} \mathrm{O} \rightleftharpoons\left(\mathrm{UO}_{2}\right)_{2} \mathrm{CO}_{3}(\mathrm{OH})_{3}^{-}+4 \mathrm{H}^{+}$ & -11.223 & 0 & 4.0 & 2 \\
\hline $\mathrm{UO}_{2}{ }^{+2}+\mathrm{SiO}_{2}(\mathrm{aq})+2 \mathrm{H}_{2} \mathrm{O} \rightleftharpoons \mathrm{UO}_{2} \mathrm{SiO}(\mathrm{OH})_{3}{ }^{+}+\mathrm{H}^{+}$ & -1.832 & 0 & 4.0 & 3 \\
\hline $\mathrm{UO}_{2}^{+2}+\mathrm{SO}_{4}^{-2} \rightleftharpoons \mathrm{UO}_{2} \mathrm{SO}_{4}(\mathrm{aq})$ & 3.0703 & 19.7626 & 3.0 & 2 \\
\hline $\mathrm{UO}_{2}{ }^{+2}+2 \mathrm{SO}_{4}^{-2} \rightleftharpoons \mathrm{UO}_{2}\left(\mathrm{SO}_{4}\right)_{2}^{-2}$ & 3.9806 & 35.6242 & 4.0 & 2 \\
\hline $\mathrm{UO}_{2}^{+2}+3 \mathrm{SO}_{4}^{-2} \rightleftharpoons \mathrm{UO}_{2}\left(\mathrm{SO}_{4}\right)_{3}^{-4}$ & 3.02 & 0 & 4.0 & 3 \\
\hline $\mathrm{UO}_{2}^{+2}+\mathrm{H}_{3} \mathrm{PO}_{4}(\mathrm{aq}) \rightleftharpoons \mathrm{UO}_{2} \mathrm{H}_{2} \mathrm{PO}_{4}^{+}+\mathrm{H}^{+}$ & 1.12 & 0 & 4.0 & 3 \\
\hline $\mathrm{UO}_{2}{ }^{+2}+\mathrm{HPO}_{4}^{-2}(\mathrm{aq}) \rightleftharpoons \mathrm{UO}_{2} \mathrm{HPO}_{4}(\mathrm{aq})$ & 7.24 & 0 & 3.0 & 3 \\
\hline $\mathrm{UO}_{2}{ }^{+2}+\mathrm{PO}_{4}^{-3} \rightleftharpoons \mathrm{UO}_{2} \mathrm{PO}_{4}^{-}$ & 13.23 & 0 & 4.0 & 3 \\
\hline $\mathrm{UO}_{2}+2+2 \mathrm{H}_{3} \mathrm{PO}_{4}(\mathrm{aq}) \rightleftharpoons \mathrm{UO}_{2}\left(\mathrm{H}_{2} \mathrm{PO}_{4}\right)_{2}(\mathrm{aq})+2 \mathrm{H}^{+}$ & 0.64 & 0 & 3.0 & 3 \\
\hline $\mathrm{UO}_{2}^{+2}+\mathrm{H}_{3} \mathrm{PO}_{4}(\mathrm{aq}) \rightleftharpoons \mathrm{UO}_{2} \mathrm{H}_{3} \mathrm{PO}_{4}^{+2}$ & 0.76 & 0 & 4.5 & 3 \\
\hline $\mathrm{UO}_{2}^{+2+}+2 \mathrm{H}_{3} \mathrm{PO}_{4}(\mathrm{aq}) \rightleftharpoons \mathrm{UO}_{2}\left(\mathrm{H}_{2} \mathrm{PO}_{4}\right)\left(\mathrm{H}_{3} \mathrm{PO}_{4}\right)^{+}+\mathrm{H}^{+}$ & 1.65 & 0 & 4.0 & 3 \\
\hline $2 \mathrm{Ca}^{+2}+\mathrm{UO}_{2}{ }^{+2}+3 \mathrm{CO}_{3}^{-2} \rightleftharpoons \mathrm{Ca}_{2} \mathrm{UO}_{2}\left(\mathrm{CO}_{3}\right)_{3}(\mathrm{aq})$ & 30.7 & 0 & 3.0 & 5 \\
\hline $\mathrm{Ca}^{+2}+\mathrm{UO}_{2}{ }^{+2}+3 \mathrm{CO}_{3}^{-2} \rightleftharpoons \mathrm{CaUO}_{2}\left(\mathrm{CO}_{3}\right)_{3}^{-2}$ & 27.18 & 0 & 4.0 & 5 \\
\hline
\end{tabular}

${ }^{\text {a }}$ Hard core diameter of species from EQ3/6's data0.ymp.R2 and data0.ymp.R4 ${ }^{1}$.

${ }^{\mathrm{b}}$ Reference: 1 Shock, et al. ${ }^{2} ; 2$ PHREEQC's llnl.dat 3; 3 Guillaumont and Mompean 4; 4 Altmaier, et al. ${ }^{5}$; and 5 Dong and Brooks ${ }^{6}$ 
Table S2. Dissolution reactions of uranium minerals

\begin{tabular}{|c|c|c|c|c|c|c|}
\hline Reaction & $\begin{array}{l}A_{0} \text { or } \\
\log K\end{array}$ & $\begin{array}{l}A_{1} \text { or } \\
\Delta_{\mathrm{r}} \mathrm{H}(\mathrm{kJ} / \mathrm{mol})\end{array}$ & $\mathbf{A}_{2}$ & $\mathbf{A}_{3}$ & $\mathbf{A}_{4}$ & $\mathbf{A}_{5}$ \\
\hline $\mathrm{UO}_{2}+4 \mathrm{H}^{+} \rightleftharpoons \mathrm{U}^{+4}+2 \mathrm{H}_{2} \mathrm{O}^{\text {a }}$ & -75.776 & -0.010558 & 5967.7 & 21.853 & 93.142 & 0 \\
\hline $\mathrm{UO}_{2}(\mathrm{am})+2 \mathrm{H}_{2} \mathrm{O} \rightleftharpoons \mathrm{U}^{+4}+4 \mathrm{OH}^{-\mathrm{b}}$ & -54.5 & 0 & & & & \\
\hline $\mathrm{UO}_{3} \cdot 2 \mathrm{H}_{2} \mathrm{O}+2 \mathrm{H}^{+} \rightleftharpoons \mathrm{UO}_{2}^{+2}+3 \mathrm{H}_{2} \mathrm{O}^{\mathrm{c}}$ & 5.35 & -50.415 & & & & \\
\hline $2 \mathrm{UO}_{2.6667}+8 \mathrm{H}^{+} \rightleftharpoons 0.6667 \mathrm{O}_{2}+2 \mathrm{U}^{+4}+4 \mathrm{H}_{2} \mathrm{O}^{\text {a }}$ & 120.95 & 0.020118 & -14968 & -53.552 & 1081300 & 0 \\
\hline $\mathrm{UO}_{2} \mathrm{CO}_{3} \rightleftharpoons \mathrm{CO}_{3}^{-2}+\mathrm{UO}_{2}^{+2 \mathrm{~d}}$ & -14.76 & -2.928 & & & & \\
\hline $\mathrm{USiO}_{4}+4 \mathrm{H}^{+} \rightleftharpoons \mathrm{SiO}_{2}+\mathrm{U}^{+4}+2 \mathrm{H}_{2} \mathrm{O}{ }^{\mathrm{e}}$ & -5.265 & -70.56 & & & & \\
\hline $\mathrm{UTi}_{2} \mathrm{O}_{6}+6 \mathrm{H}_{2} \mathrm{O} \rightleftharpoons \mathrm{U}(\mathrm{OH})_{4}+2 \mathrm{Ti}(\mathrm{OH})_{4}{ }^{\mathrm{f}}$ & -33.511 & 39.013 & & & & \\
\hline $\mathrm{Ca}\left(\mathrm{UO}_{2}\right)_{2}\left(\mathrm{PO}_{4}\right)_{3} \cdot 3 \mathrm{H}_{2} \mathrm{O} \rightleftharpoons \mathrm{Ca}^{+2}+2 \mathrm{UO}_{2}+2+2 \mathrm{PO}_{4}^{-3}$ & -48.36 & 0 & & & & \\
\hline$+3 \mathrm{H}_{2} \mathrm{Og}$ & & & & & & \\
\hline $\begin{array}{l}\mathrm{Ca}\left(\mathrm{UO}_{2}\right)_{2}\left(\mathrm{SiO}_{3} \mathrm{OH}\right)_{2} \cdot 5 \mathrm{H}_{2} \mathrm{O}+6 \mathrm{H}^{+} \rightleftharpoons \mathrm{Ca}^{+2+} \\
2 \mathrm{UO}_{2}^{+2}+2 \mathrm{SiO}_{2}+9 \mathrm{H}_{2} \mathrm{O}^{\mathrm{h}}\end{array}$ & 10.71 & -109.87 & & & & \\
\hline
\end{tabular}

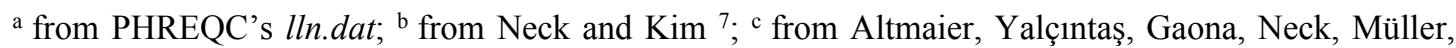
Schlieker and Fanghänel 5; d from Guillaumont and Mompean 4; e from Szenknect, et al. ${ }^{8 ;}{ }^{8}$ from Donaldson, et al. ${ }^{9} ; \mathrm{g}$ from Gorman-Lewis, et al. ${ }^{10}$; and ${ }^{\mathrm{h}}$ from Shvareva, et al. ${ }^{11}$.

Table S3. Redox reactions of uranium from PHREEQC's llnl.dat

\begin{tabular}{lllllll}
\hline Reaction & A0 & A1 & A2 & A3 & A4 & A5 \\
\hline $\mathrm{UO}_{2}{ }^{+2}+2 \mathrm{H}^{+} \rightleftharpoons \mathrm{U}^{+4}+0.5 \mathrm{O}_{2}(\mathrm{aq})+\mathrm{H}_{2} \mathrm{O}$ & 44.4837 & 0.010129 & -11787 & -19.194 & 464360 & 0 \\
\hline
\end{tabular}

Table S4. Other related reactions from PHREEQC's Ilnl.dat

\begin{tabular}{|c|c|}
\hline Complexation Reactions & Dissolution Reactions \\
\hline $\mathrm{H}_{2} \mathrm{O} \rightleftharpoons \mathrm{H}_{2}(\mathrm{aq})+0.5 \mathrm{O}_{2}(\mathrm{aq})$ & $\mathrm{H}_{2}(\mathrm{~g}) \rightleftharpoons \mathrm{H}_{2}(\mathrm{aq})^{\mathrm{a}}$ \\
\hline $\mathrm{H}_{2} \mathrm{O} \rightleftharpoons \mathrm{OH}^{-}+\mathrm{H}^{+}$ & $\mathrm{O}_{2}(\mathrm{~g}) \rightleftharpoons \mathrm{O}_{2}(\mathrm{aq})^{\mathrm{a}}$ \\
\hline $\mathrm{HCO}_{3}^{-}+\mathrm{H}^{+} \rightleftharpoons \mathrm{CO}_{2}(\mathrm{aq})+\mathrm{H}_{2} \mathrm{O}$ & $\mathrm{N}_{2}(\mathrm{~g}) \rightleftharpoons \mathrm{N}_{2}(\mathrm{aq})^{\mathrm{a}}$ \\
\hline $\mathrm{HCO}_{3}^{-} \rightleftharpoons \mathrm{CO}_{3}^{-2}+\mathrm{H}^{+}$ & $\mathrm{CO}_{2}(\mathrm{~g}) \rightleftharpoons \mathrm{CO}_{2}(\mathrm{aq})^{\mathrm{a}}$ \\
\hline $\mathrm{HCO}_{3}{ }^{-}+\mathrm{Ca}^{+2} \rightleftharpoons \mathrm{CaCO}_{3}(\mathrm{aq})+\mathrm{H}^{+}$ & $\mathrm{SiO}_{2}(\mathrm{Quartz}) \rightleftharpoons \mathrm{SiO}_{2}(\mathrm{aq})$ \\
\hline $\mathrm{HCO}_{3}{ }^{-}+\mathrm{Ca}^{+2} \rightleftharpoons \mathrm{CaHCO}_{3}^{+}$ & $\mathrm{KAlSi}_{3} \mathrm{O}_{8}(\mathrm{~K}$-Feldspar $)+4 \mathrm{H}^{+} \rightleftharpoons \mathrm{Al}^{+3}+\mathrm{K}^{+}+\mathrm{H}_{2} \mathrm{O}+3 \mathrm{SiO}_{2}(\mathrm{aq})$ \\
\hline $\mathrm{HPO}_{4}^{-2}+\mathrm{H}^{+} \rightleftharpoons \mathrm{H}_{2} \mathrm{PO}_{4}^{-}$ & $\mathrm{CaCO}_{3}($ Calcite $)+\mathrm{H}^{+} \rightleftharpoons \mathrm{Ca}^{+2}+\mathrm{HCO}_{3}^{-}$ \\
\hline $\mathrm{HPO}_{4}^{-2}+2 \mathrm{H}^{+} \rightleftharpoons \mathrm{H}_{3} \mathrm{PO}_{4}(\mathrm{aq})$ & $\mathrm{FeS}_{2}($ Pyrite $)+\mathrm{H}_{2} \mathrm{O} \rightleftharpoons 0.25 \mathrm{H}^{+}+0.25 \mathrm{SO}_{4}^{-2}+\mathrm{Fe}^{+2}+1.75 \mathrm{HS}^{-}$ \\
\hline $\mathrm{SO}_{4}^{-2}+\mathrm{H}^{+} \rightleftharpoons \mathrm{HSO}_{4}^{-}$ & $\mathrm{Fe}_{2} \mathrm{O}_{3}($ Hematite $)+6 \mathrm{H}^{+} \rightleftharpoons 2 \mathrm{Fe}^{+3}+3 \mathrm{H}_{2} \mathrm{O}$ \\
\hline $\mathrm{SO}_{4}^{-2}+\mathrm{Ca}^{+2} \rightleftharpoons \mathrm{CaSO}_{4}(\mathrm{aq})$ & $\mathrm{NaAlSi}_{3} \mathrm{O}_{8}($ Albite $)+4 \mathrm{H}^{+} \rightleftharpoons \mathrm{Al}^{+3}+\mathrm{Na}^{+}+2 \mathrm{H}_{2} \mathrm{O}+3 \mathrm{SiO}_{2}(\mathrm{aq})$ \\
\hline $\mathrm{SO}_{4}^{-2}+\mathrm{Al}^{+3} \rightleftharpoons \mathrm{AlSO}_{4}^{+}$ & $\mathrm{CaSO}_{4}($ Anhydrite $) \rightleftharpoons \mathrm{Ca}^{+2}+\mathrm{SO}_{4}^{-2}$ \\
\hline $2 \mathrm{SO}_{4}^{-2}+\mathrm{Al}^{+3} \rightleftharpoons \mathrm{Al}\left(\mathrm{SO}_{4}\right)_{2}^{-}$ & $\mathrm{NaCl}($ Halite $) \rightleftharpoons \mathrm{Cl}^{-}+\mathrm{Na}^{+}$ \\
\hline \multicolumn{2}{|l|}{$\mathrm{SO}_{4}^{-2}+\mathrm{Fe}^{+2} \rightleftharpoons \mathrm{FeSO} 4(\mathrm{aq})$} \\
\hline \multicolumn{2}{|l|}{$\mathrm{SO}_{4}^{-2}+\mathrm{H}^{+} \rightleftharpoons \mathrm{HS}^{-}+2 \mathrm{O}_{2}(\mathrm{aq})$} \\
\hline \multicolumn{2}{|l|}{$\mathrm{Fe}^{+2}+0.25 \mathrm{O}_{2}(\mathrm{aq})+\mathrm{H}^{+} \rightleftharpoons \mathrm{Fe}^{+3}+0.5 \mathrm{H}_{2} \mathrm{O}$} \\
\hline $\mathrm{Fe}^{+3}+2 \mathrm{H}_{2} \mathrm{O} \rightleftharpoons \mathrm{Fe}(\mathrm{OH})_{2}^{+}+2 \mathrm{H}^{+}$ & \\
\hline $\mathrm{Fe}^{+3}+\mathrm{H}_{2} \mathrm{O} \rightleftharpoons \mathrm{FeOH}^{+2}+\mathrm{H}^{+}$ & \\
\hline
\end{tabular}

a The parameters of critical temperature, critical pressure and acentric factor are selected from PHREEQC's phreeqc.dat.

Table S5. Solubility data of uranium minerals

\begin{tabular}{llllll}
\hline Reference & Mineral & Solution & pH & Temperature/K & Pressure/atm(gas) \\
\hline Parks and Pohl ${ }^{12}$ & $\mathrm{UO}_{2}(\mathrm{cr})$ & $<0.1 \mathrm{M} \mathrm{NaCl}$ & $1-10$ & $373.15-573.15$ & $493.46\left(\mathrm{H}_{2}\right)$ \\
Torrero, et al. ${ }^{13}$ & $\mathrm{UO}_{2}(\mathrm{cr})$ & $0.008 \mathrm{M}$ & $0.66-12$ & 298.15 & $1\left(\mathrm{H}_{2}\right)$ \\
& & $\mathrm{NaClO}_{4} / 1 \mathrm{M} \mathrm{NaCl}$ & & &
\end{tabular}




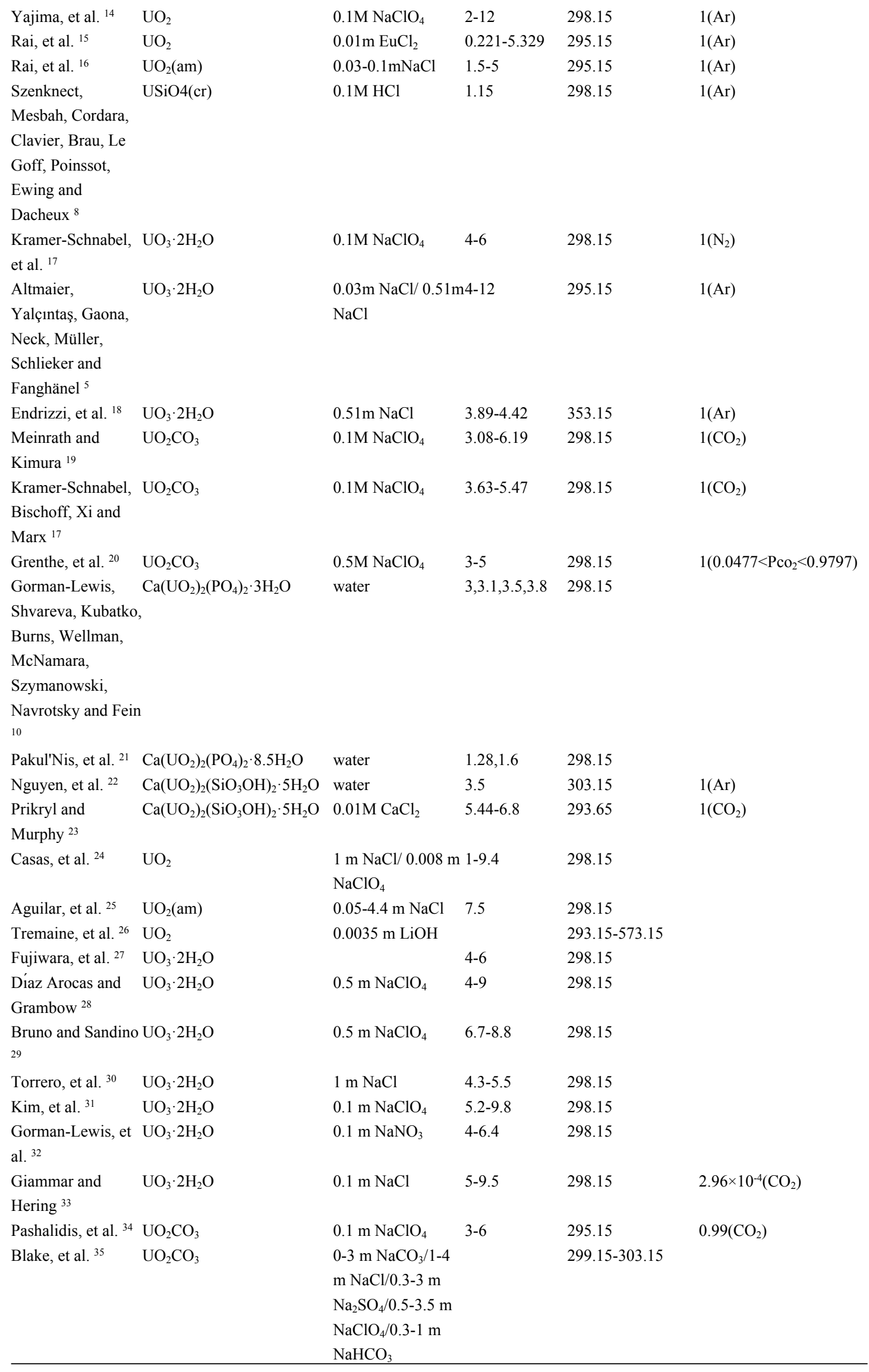




\section{Reference}

1. Daveler, S. A.; Wolery, T. J. EQPT, a data file preprocessor for the EQ3/6 software package: user's guide and related documentation (version 7.0); Lawrence Livermore National Lab: 1992.

2. Shock, E. L.; Sassani, D. C.; Betz, H., Uranium in geologic fluids: Estimates of standard partial molal properties, oxidation potentials, and hydrolysis constants at high temperatures and pressures. Geochim. Cosmochim. Acta 1997, 61, 4245-4266.

3. Parkhurst, D. L.; Appelo, C. Description of input and examples for PHREEQC version 3: a computer program for speciation, batch-reaction, one-dimensional transport, and inverse geochemical calculations; 2328-7055; US Geological Survey: 2013.

4. Guillaumont, R.; Mompean, F. J., Update on the chemical thermodynamics of uranium, neptunium, plutonium, americium and technetium. Elsevier Amsterdam: 2003; Vol. 5.

5. Altmaier, M.; Yalçıntaş, E.; Gaona, X.; Neck, V.; Müller, R.; Schlieker, M.; Fanghänel, T., Solubility of U(VI) in chloride solutions. I. The stable oxides/hydroxides in $\mathrm{NaCl}$ systems, solubility products, hydrolysis constants and SIT coefficients. J. Chem. Thermodyn. 2017, 114, 2-13.

6. Dong, W.; Brooks, S. C., Determination of the Formation Constants of Ternary Complexes of Uranyl and Carbonate with Alkaline Earth Metals $(\mathrm{Mg} 2+, \mathrm{Ca} 2+, \mathrm{Sr} 2+$, and $\mathrm{Ba} 2+)$ Using Anion Exchange Method. Environ. Sci. Technol. 2006, 40, 4689-4695.

7. Neck, V.; Kim, J. I., Solubility and hydrolysis of tetravalent actinides. Radiochim. Acta 2001, 89, $1-16$.

8. Szenknect, S.; Mesbah, A.; Cordara, T.; Clavier, N.; Brau, H.-P.; Le Goff, X.; Poinssot, C.; Ewing, R. C.; Dacheux, N., First experimental determination of the solubility constant of coffinite. Geochim. Cosmochim. Acta 2016, 181, 36-53.

9. Donaldson, M. H.; Stevens, R.; Lang, B. E.; Boerio-Goates, J.; Woodfield, B. F.; Putnam, R. L.; Navrotsky, A., Heat capacities and absolute entropies of UTi2O6 and CeTi2O6. J. Therm. Anal. Calorim. 2005, 81, 617-625.

10. Gorman-Lewis, D.; Shvareva, T.; Kubatko, K. A.; Burns, P. C.; Wellman, D. M.; McNamara, B.; Szymanowski, J. E.; Navrotsky, A.; Fein, J. B., Thermodynamic properties of autunite, uranyl hydrogen phosphate, and uranyl orthophosphate from solubility and calorimetric measurements. Environ. Sci. Technol. 2009, 43, 7416-22.

11. Shvareva, T. Y.; Mazeina, L.; Gorman-Lewis, D.; Burns, P. C.; Szymanowski, J. E. S.; Fein, J. B.; Navrotsky, A., Thermodynamic characterization of boltwoodite and uranophane: Enthalpy of formation and aqueous solubility. Geochim. Cosmochim. Acta 2011, 75, 5269-5282.

12. Parks, G. A.; Pohl, D. C., Hydrothermal solubility of uraninite. Geochim. Cosmochim. Acta 1988, $52,863-875$.

13. Torrero, M. E.; Casas, I.; Aguilar, M.; De Pablo, J.; Gimenez, J.; Bruno, J., The Solubility of Unirradiated UO2 In Both Perchlorate And Chloride Test Solutions. Influence of the Ionic Medium. MRS Proc. 2011, 212, 229-234.

14. Yajima, T.; Kawamura, Y.; Ueta, S., Uranium(IV) Solubility and Hydrolysis Constants Under Reduced Conditions. MRS Proc. 2011, 353, 1137-1142.

15. Rai, D.; Yui, M.; Moore, D. A., Solubility and Solubility Product at $22^{\circ} \mathrm{C}$ of UO2(c) Precipitated 
from Aqueous U(IV) Solutions. J. Solution Chem. 2003, 32, 1-17.

16. Rai, D.; Felmy, A. R.; Sterner, S. M.; Moore, D. A.; Mason, M. J.; Novak, C. F., The solubility of $\mathrm{Th}(\mathrm{IV})$ and $\mathrm{U}(\mathrm{IV})$ hydrous oxides in concentrated $\mathrm{NaCl}$ and $\mathrm{MgCl} 2$ solutions. Radiochim. Acta 1997, 79, 239-247.

17. Kramer-Schnabel, U.; Bischoff, H.; Xi, R. H.; Marx, G., Solubility Products and Complex Formation Equilibria in the Systems Uranyl Hydroxide and Uranyl Carbonate at $25^{\circ} \mathrm{C}$ and $\mathrm{I}=0.1 \mathrm{M}$. Radiochim. Acta 1992, 56, 183-188.

18. Endrizzi, F.; Gaona, X.; Marques Fernandes, M.; Baeyens, B.; Altmaier, M., Solubility and hydrolysis of $\mathrm{U}(\mathrm{VI})$ in $0.5 \mathrm{~mol} / \mathrm{kg} \mathrm{NaCl}$ solutions at $\mathrm{T}=22$ and $80^{\circ} \mathrm{C}$. J. Chem. Thermodyn. 2018, 120 , 45-53.

19. Meinrath, G.; Kimura, T., Carbonate complexation of the uranyl(VI) ion. J. Alloys Compd. 1993, 202, 89-93.

20. Grenthe, I.; Ferri, D.; Salvatore, F.; Riccio, G., Studies on metal carbonate equilibria. Part 10. A solubility study of the complex formation in the uranium(VI)-water-carbon dioxide (g) system at $25^{\circ} \mathrm{C}$. J. Chem. Soc., Dalton Trans. 1984, 2439-2443.

21. Pakul'Nis, G.; Shmariovich, Y. M.; Zhil'Tsova, I.; Polupanova, L.; Shugina, G., Mineral assemblages and physicochemical conditions for carnotite-autunite-torbernite mineralization in carbonaceous phyllites. Int. Geol. Rev. 1986, 28, 100-113.

22. Nguyen, S. N.; Silva, R. J.; Weed, H. C.; Andrews, J. E., Standard Gibbs free energies of formation at the temperature $303.15 \mathrm{~K}$ of four uranyl silicates: soddyite, uranophane, sodium boltwoodite, and sodium weeksite. J. Chem. Thermodyn. 1992, 24, 359-376.

23. Prikryl, J. D.; Murphy, W. M. In The solubility of uranophane under oxidizing conditions in $\mathrm{CaCl} 2$ and $\mathrm{SiO} 2$ (aq) test solutions, 8th International Conference on the Chemistry and Migration Behavior of Actinides and Fission Products in the Geosphere, Migration '01, Bregenz, Austria, 2001; 2001.

24. Casas, I.; de Pablo, J.; Giménez, J.; Torrero, M. E.; Bruno, J.; Cera, E.; Finch, R. J.; Ewing, R. C., The role of pe, $\mathrm{pH}$, and carbonate on the solubility of $\mathrm{UO} 2$ and uraninite under nominally reducing conditions. Geochim. Cosmochim. Acta 1998, 62, 2223-2231.

25. Aguilar, M.; Casas, I.; Depablo, J.; Torrero, M. E., Effect of Chloride Concentration on the Solubility of Amorphous Uranium-Dioxide at 25-Degrees C under Reducing Conditions. Radiochim. Acta 1991, 52-3, 13-15.

26. Tremaine, P. R.; Chen, J. D.; Wallace, G. J.; Boivin, W. A., Solubility of uranium (IV) oxide in alkaline aqueous solutions to 300C. J. Solution Chem. 1981, 10, 221-230.

27. Fujiwara, K.; Yamana, H.; Fujii, T.; Kawamoto, K.; Sasaki, T.; Moriyama, H., Solubility Product of Hexavalent Uranium Hydrous Oxide. J. Nucl. Sci. Technol. 2005, 42, 289-294.

28. Díaz Arocas, P.; Grambow, B., Solid-liquid Phase Equilibria of U(VI) in $\mathrm{NaCl}$ Solutions. Geochim. Cosmochim. Acta 1998, 62, 245-263.

29. Bruno, J.; Sandino, A., The Solubility of Amorphous and Crystalline Schoepite in Neutral to Alkaline Aqueous Solutions. MRS Proc. 1988, 127, 871.

30. Torrero, M. E.; Casas, I.; Pablo, J. d.; Sandino, M. C. A.; Grambow, B., A Comparison Between Unirradiated UO2(s) and Schoepite Solubilities in $1 \mathrm{M} \mathrm{NaCl}$ Medium. Radiochim. Acta 1994, 66-67, 2936.

31. Kim, W. H.; Choi, K. C.; Park, K. K.; Eom, T. Y., Effects of Hypochlorite Ion on the Solubility of Amorphous Schoepite at $25^{\circ} \mathrm{C}$ in Neutral to Alkaline Aqueous Solutions. Radiochim. Acta 1994, 66-67, 45-50. 
32. Gorman-Lewis, D.; Fein, J. B.; Burns, P. C.; Szymanowski, J. E. S.; Converse, J., Solubility measurements of the uranyl oxide hydrate phases metaschoepite, compreignacite, Na-compreignacite, becquerelite, and clarkeite. J. Chem. Thermodyn. 2008, 40, 980-990.

33. Giammar, D. E.; Hering, J. G., Influence of Dissolved Sodium and Cesium on Uranyl Oxide Hydrate Solubility. Environ. Sci. Technol. 2004, 38, 171-179.

34. Pashalidis, I.; Czerwinski, K. R.; Fanghanel, T.; Kim, J. I., Solid-liquid phase equilibria of Pu(VI) and U(VI) in aqueous carbonate systems. Determination of stability constants. Radiochim. Acta 1997, 76, 55-62.

35. Blake, C. A.; Coleman, C. F.; Brown, K. B.; Hill, D. G.; Lowrie, R. S.; Schmitt, J. M., Studies in the Carbonate-Uranium System. J. Am. Chem. Soc. 1956, 78, 5978-5983. 